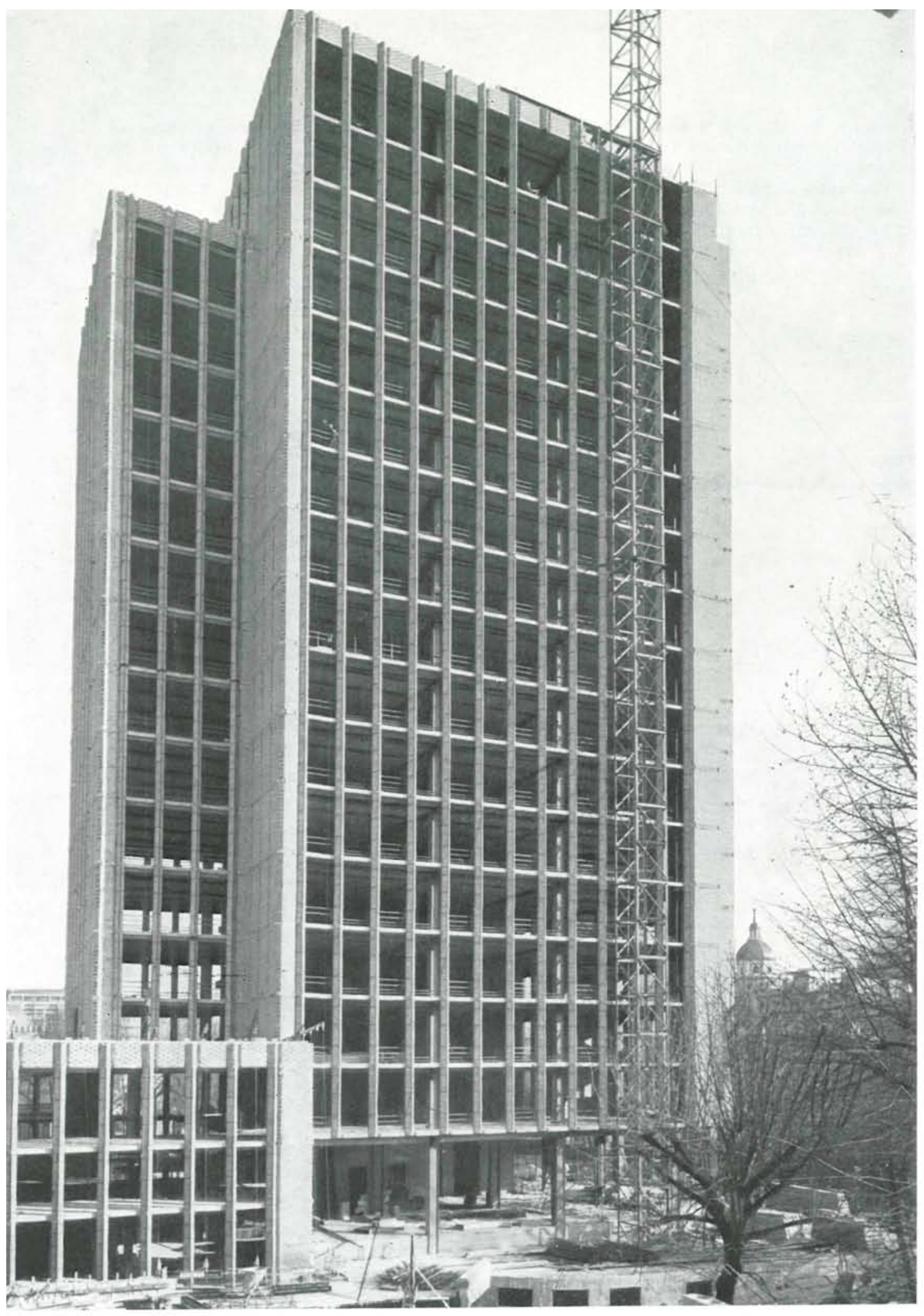

\title{
la construcción del edificio para "La Unión y El Fénix Español", en Madrid - España
}

L. GUTIERREZ SOTO, arquitecto

J. M. ${ }^{\circ}$ SAENZ DE BENITO, V. URQUIA, J. GASCON y MARIN y J. A. FERNANDEZ DURAN, ingenieros simomsis Se explican en este artículo los detalles más interesantes de su construcción, los problemas que ha presentado la cimentación - dadas las características especiales del terreno- y las soluciones adoptadas; asimismo se describe la especial realización del núcleo central de ascensores, con su arranque metálico y el resto del fuste ejecutado con encofrado deslizante. El edificio tiene tres sótanos y 19 plantas útiles. 
En el Paseo de la Castellana, de Madrid, y para Sede Social de "La Unión y El Fénix Español», se está construyendo un gran edificio, según proyecto y bajo dirección técnica del arquitecto D. Luis Gutiérrez Soto. El edificio, con un total aproximado de $28.000 \mathrm{~m}^{2}$, tiene tres sótanos y 19 plantas útiles. De los tres sótanos, los dos inferiores, dedicados a aparcamiento para una cabida total de 225 vehículos, y el superior, destinado a archivos. Los accesos al aparcamiento se hacen directamente desde el jardín, por medio de dos rampas, una de entrada y otra de salida.

sección por los

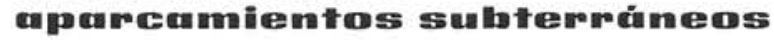

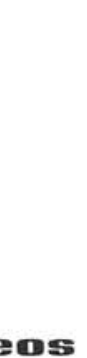

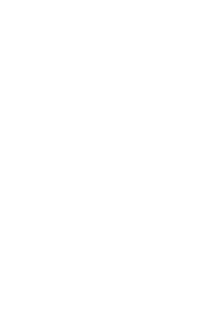

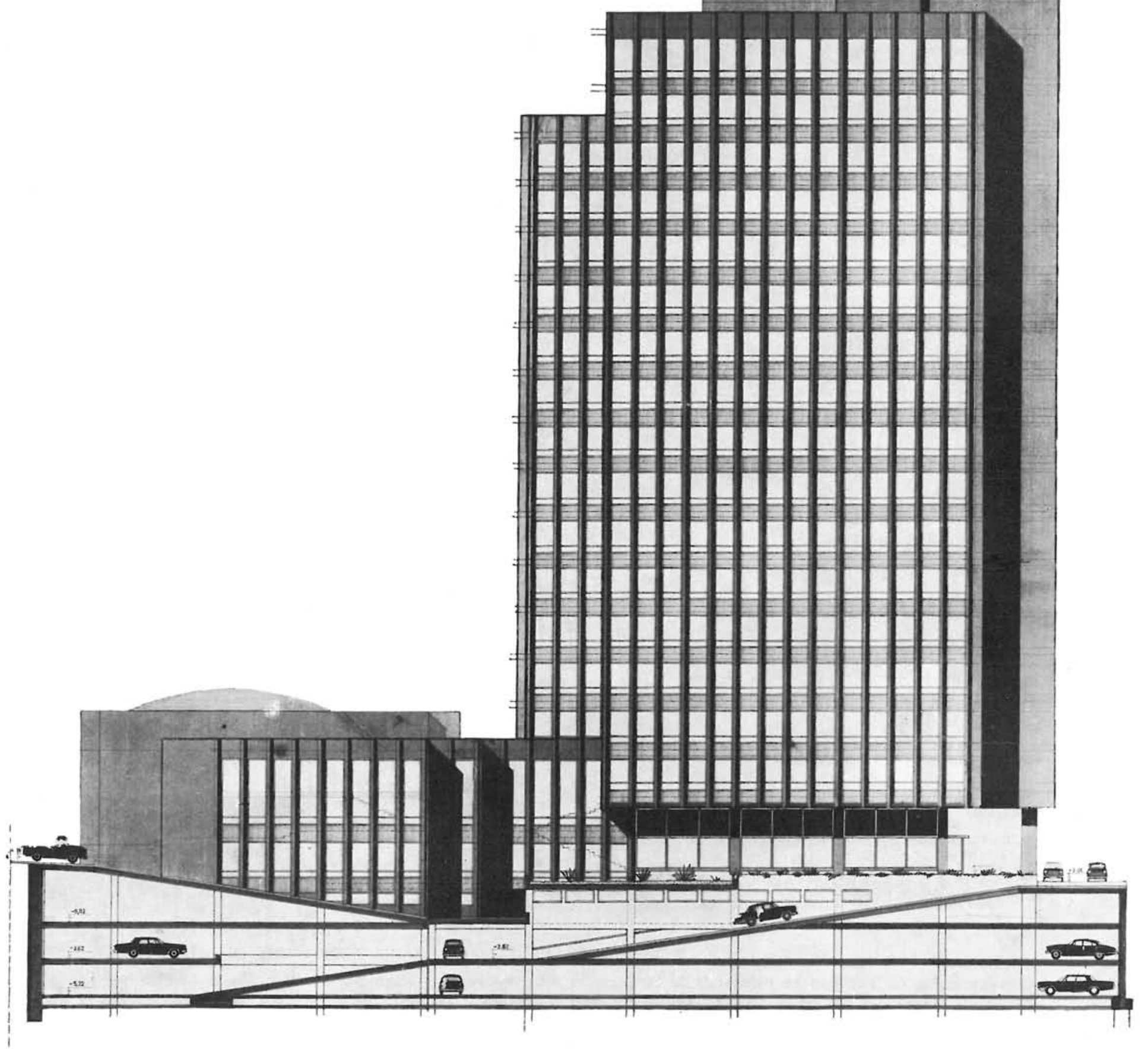




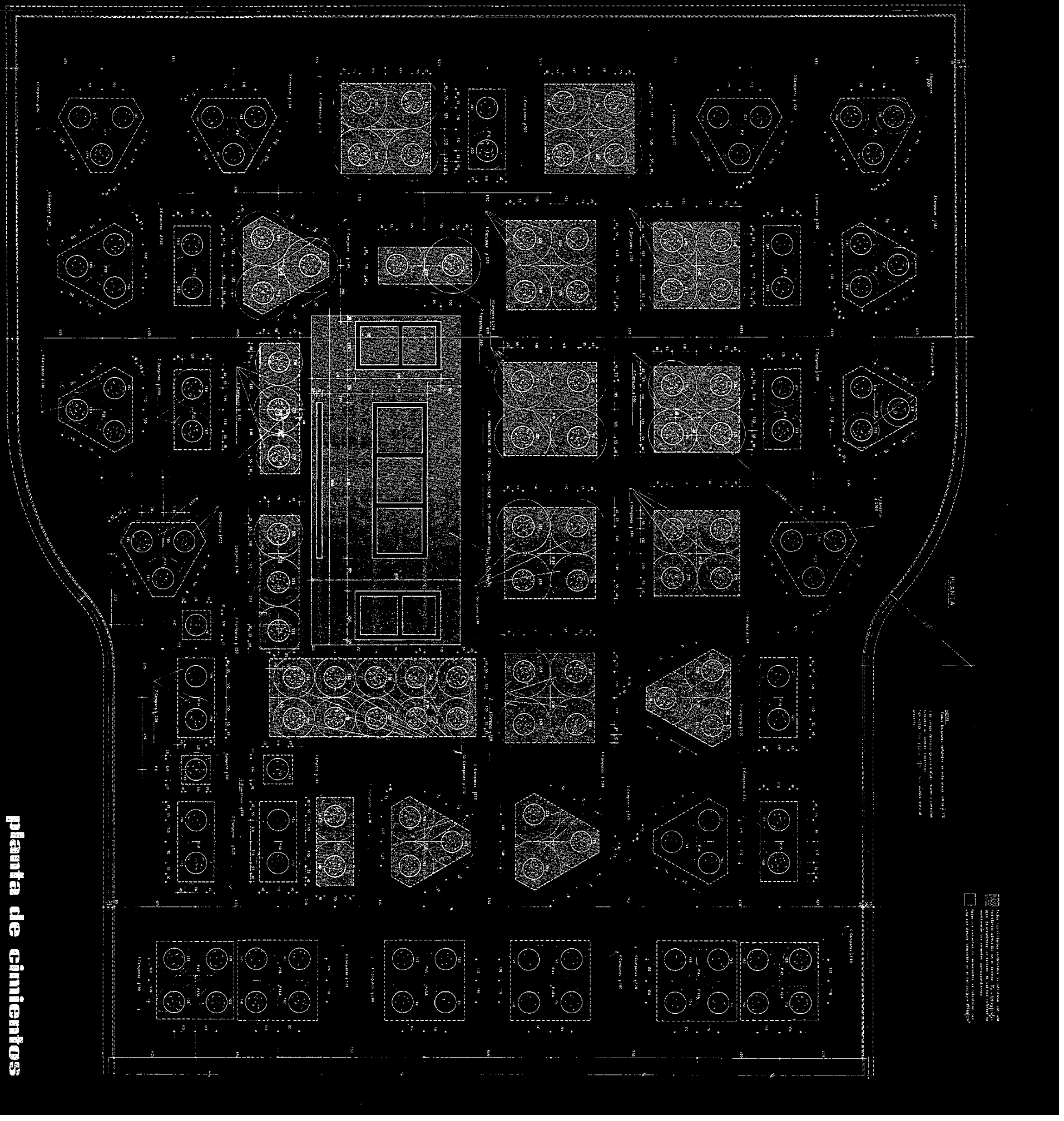



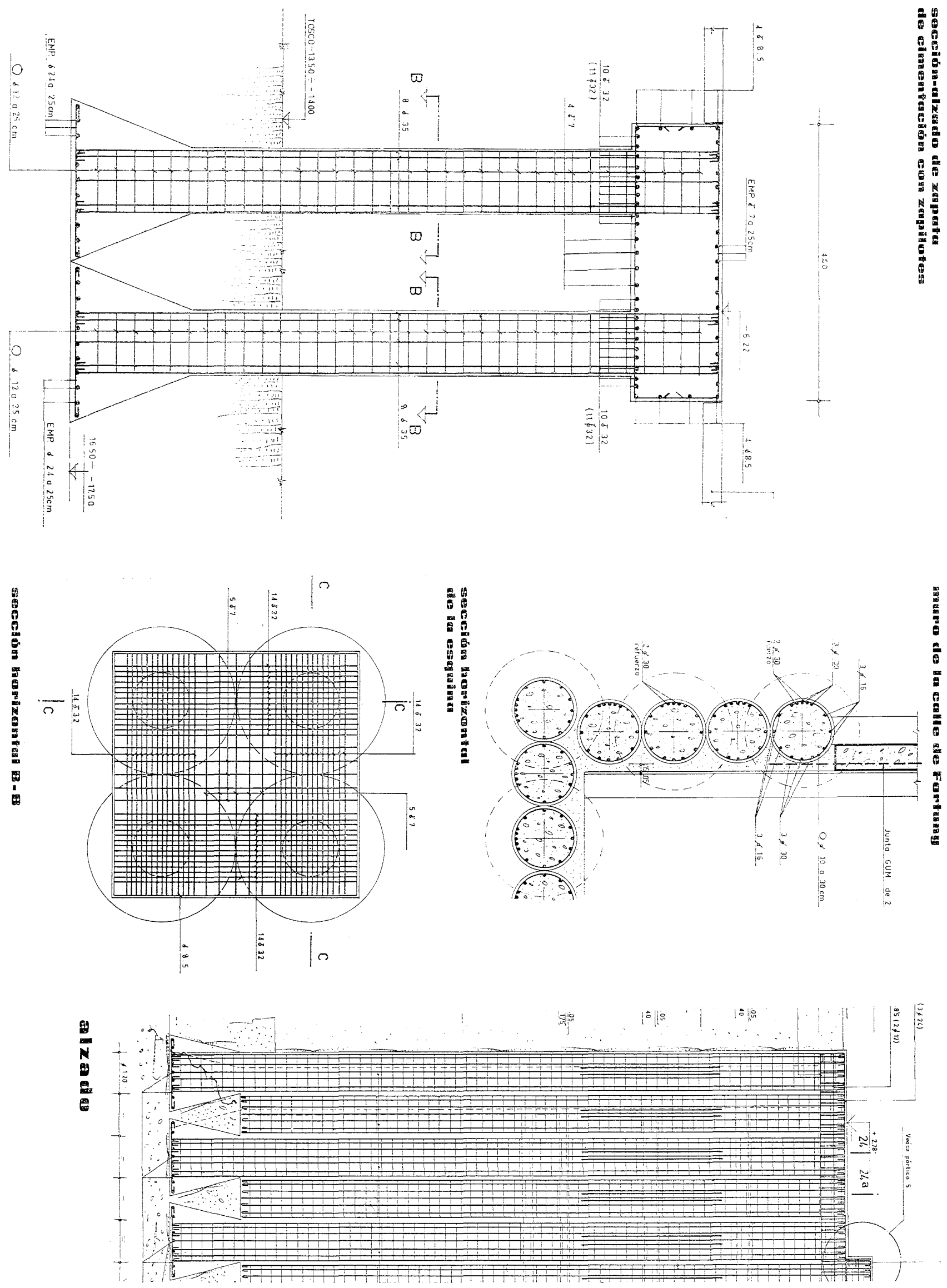
Siendo la Castellana el lecho de un antiguo río, casi todo su terreno es malo para cimentar, ya que se encuentran muchas capas de arena fina con capas intermedias de arcilla con gran cantidad de agua, que hacen imposible cimentar sobre estos estratos grandes cargas.

Estando comprendidas las cargas de los pilares entre 1.000 a $1.400 \mathrm{t}$, se empleó el sistema de cimentación por zapilotes de $1,15 \mathrm{~m}$ de diámetro, ensanchados en su base hasta $2,80 \mathrm{~m}$ de diámetro, a fin de que sus cargas admisibles fuesen sensiblemente mayores, ya que para el cálculo se consideraba nula la acción por fuste, debido a las grandes cantidades de agua existentes en el terreno. Los zapilotes tenían una profundidad de unos $11,00 \mathrm{~m}$ desde el nivel del último sótano y penetraban unos $2 \mathrm{~m}$ en el "tosco", terreno arcilloso impermeable, para el que se tomó una resistencia de $6 \mathrm{~kg} / \mathrm{cm}^{2}$ después de una serie de ensayos. En total se ejecutaron 286 pilotes.

Junto a la calle de Fortuny, como los sótanos llegaban hasta el límite del solar, fue necesario ejecutar un muro pantalla a base de pilotes tangentes, los cuales, alternativamente, se ensanchaban como los zapilotes. En todos ellos, y a cota correspondiente a los forjados de la estructura, se dejaron unas virolas metálicas soldadas a la armadura, para, a su vez, soldar posteriormente a ellas las vigas de los pisos. Todo el trabajo de pilotaje y tablestacado fue ejecutado por la firma KRONSA.

El núcleo central, donde están situados los cinco ascensores del edificio, se ejecutó en hormigón armado para que, de esta forma, funcionando como una viga en

\section{SERECín verpical}
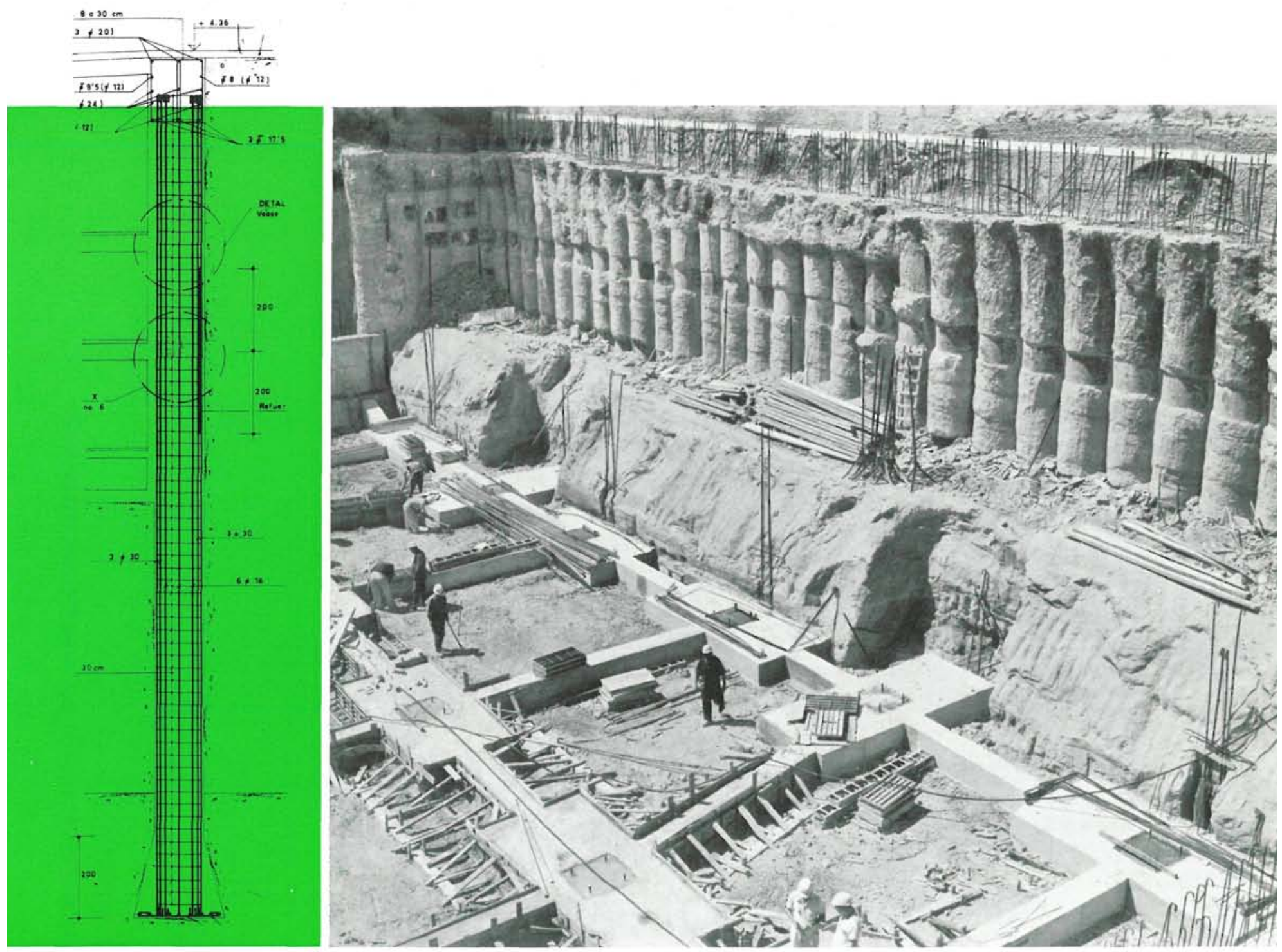

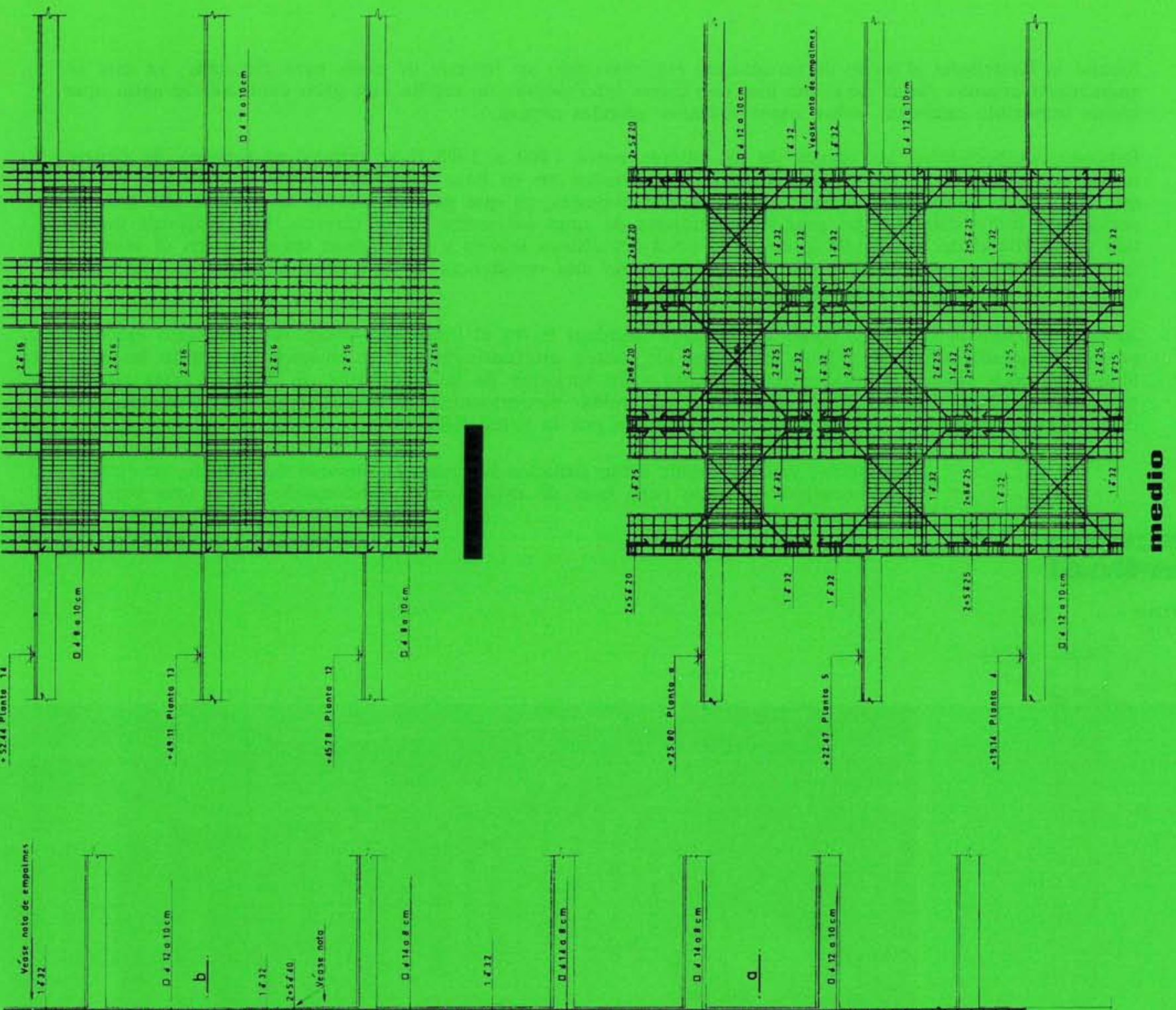

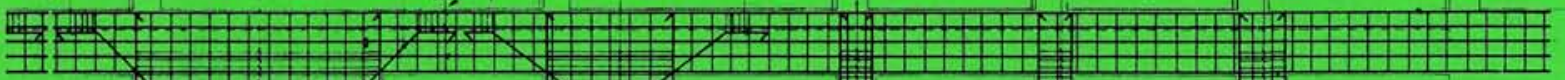

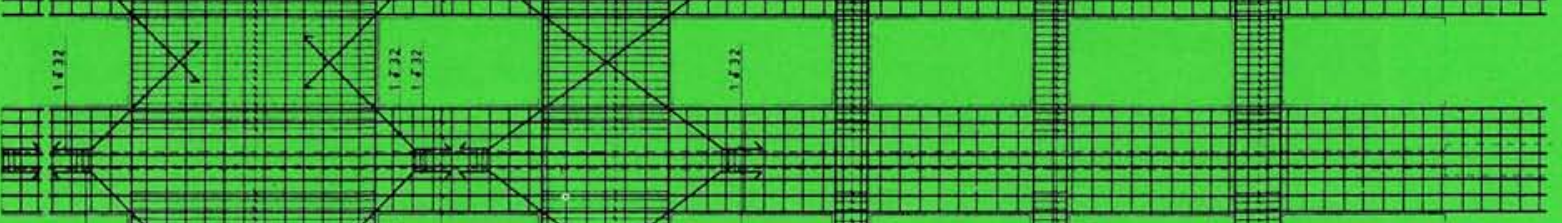

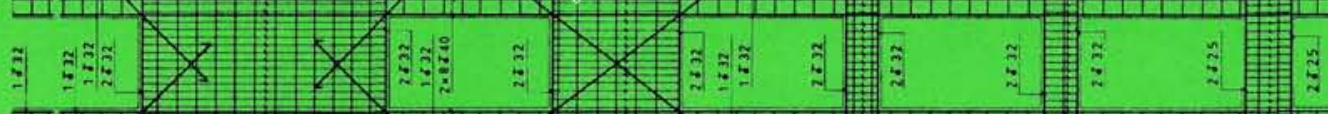
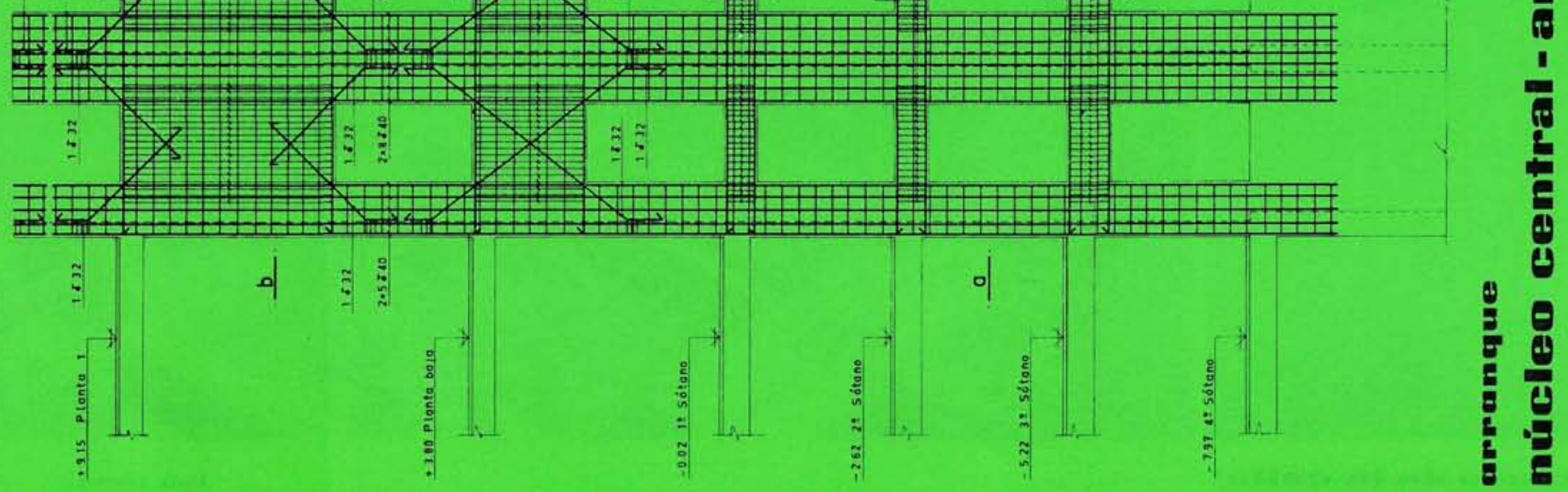
foriendas alle sótramos y currotanture allel

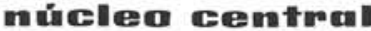
en pallasstron
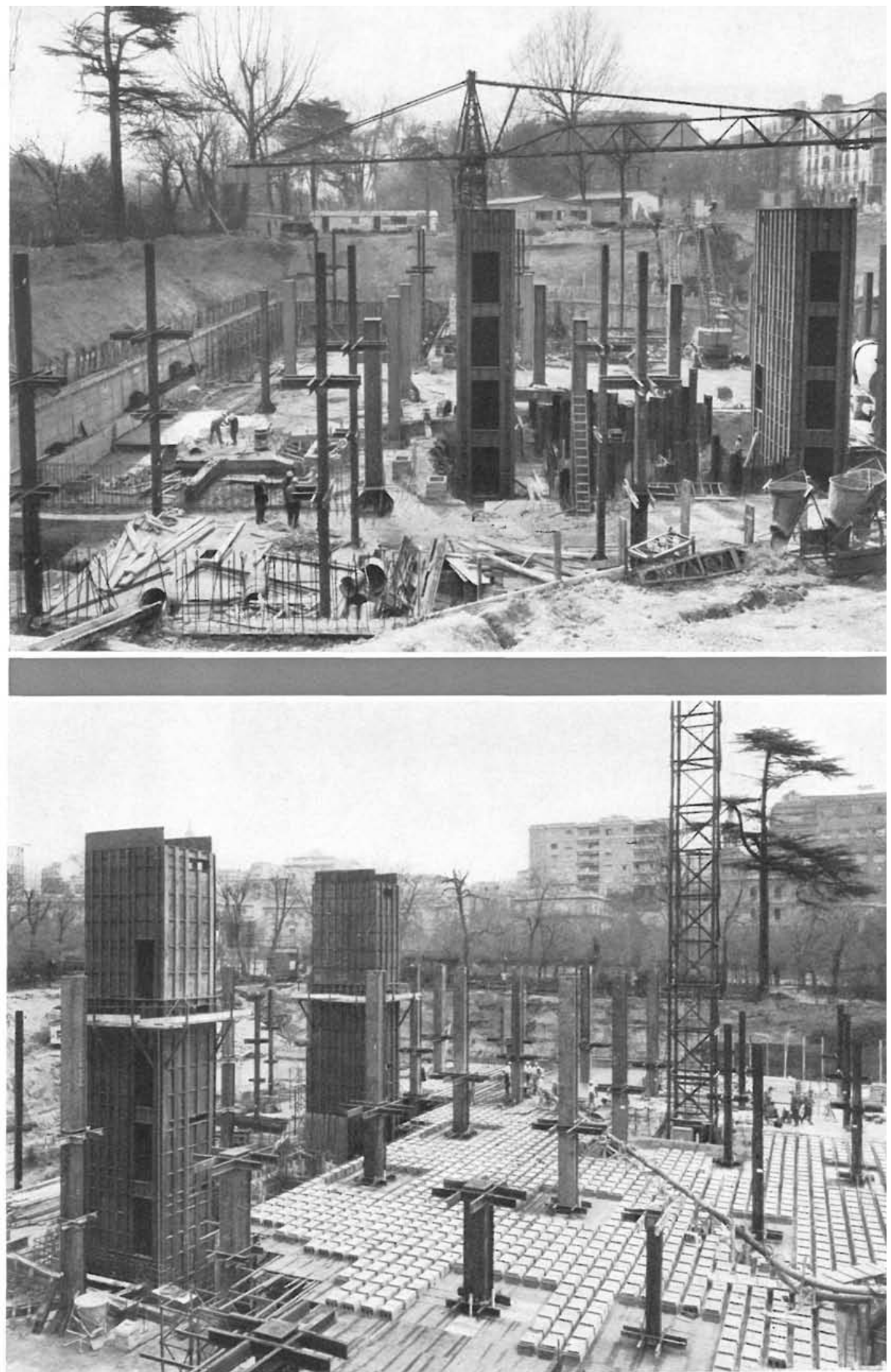

ménsula, absorbiese prácticamente el $90 \%$ de los esfuerzos de viento, ya que su inercia es mucho mayor que la de los pilares y éstos, por lo tanto, trabajan casi exclusivamente a compresión.

Esta viga en ménsula trabaja apoyada libremente sobre la cimentación y apoyada horizontalmente en la solera armada del tercer sótano y de los forjados del tercer y segundo sótano, a partir de cuyo nivel funciona como voladizo hasta su cota máxima. Las reacciones horizontales se transmiten por medio de los forjados y la solera, mencionados anteriormente, a los muros perimetrales de hormigón armado, que, a su vez, lo transmiten al terreno. 


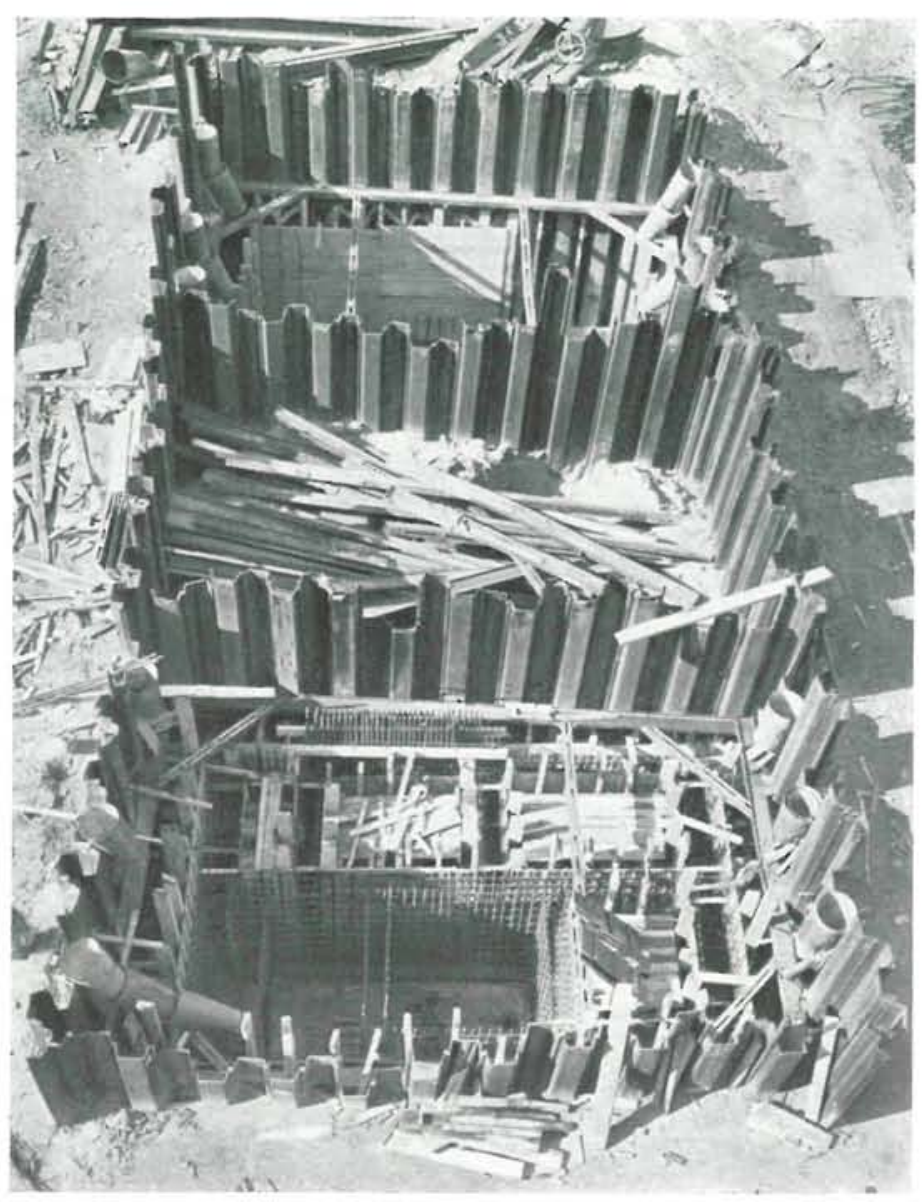

Núcleo central: fase de tablestacado.

No se ha considerado que el forjado del sótano primero transmita ningún esfuerzo, debido a la posibilidad de que en su día se abran zanjas junto a los muros para nuevos servicios que puedan ocasionar algún quebranto. Estos forjados de los sótanos se hicieron del tipo sin vigas. La carga vertical de todo el núcleo de hormigón armado, descrito anteriormente, es de unas $7.400 \mathrm{t}$, y el momento máximo en el forjado del primer sótano es de $11.700 \mathrm{~m} \cdot \mathrm{t}$.

La cimentación de este núcleo hubo que bajarla también hasta el tosco y para ello se utilizaron tres recintos de tablestacas. Dicha cimentación se hizo a base de recintos huecos formados por muros y forjados de hormigón para evitar que aumentase el peso propio de la misma.

Debido al gran momento de $11.700 \mathrm{~m} \cdot \mathrm{Mp}$ fue necesario hacer toda la parte inferior de los dos elementos laterales del núcleo central en palastro, ya que se quisieron mantener unos espesores máximos de $25 \mathrm{~cm}$ en los muros de hormigón para evitar sensación de pesadez en la entrada de los ascensores.

A causa de que la situación del núcleo no coincidía con el centro de gravedad de la planta, al actuar los esfuerzos de viento se producían unas torsiones, y para conseguir una efectiva colaboración de los pórticos con el núcleo central,

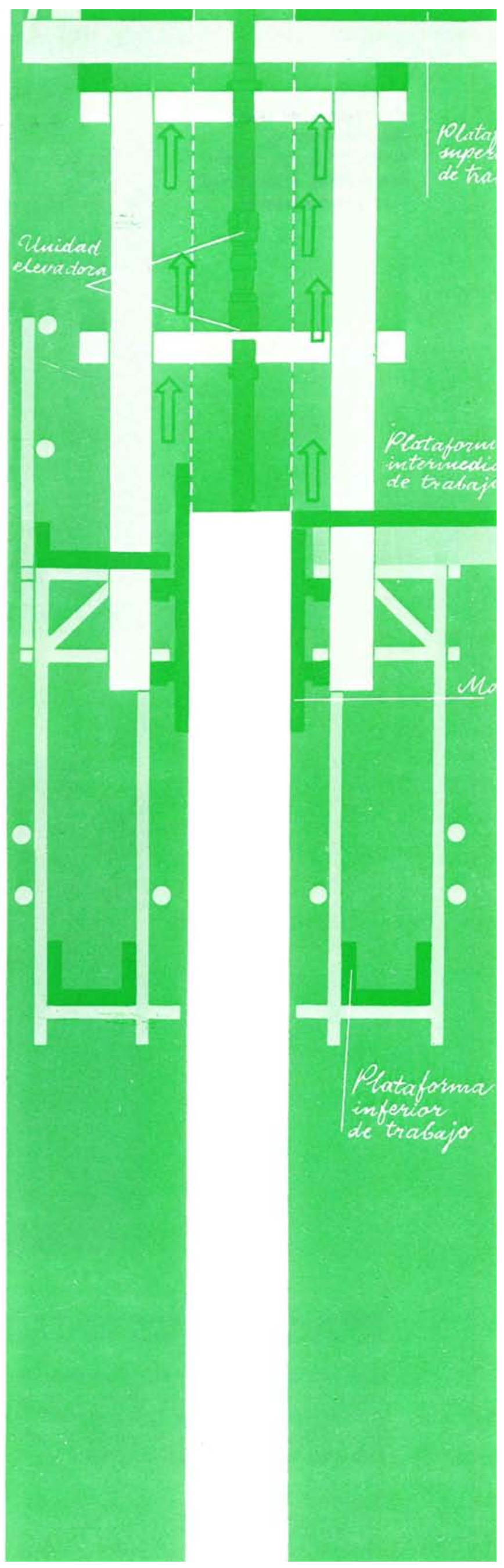



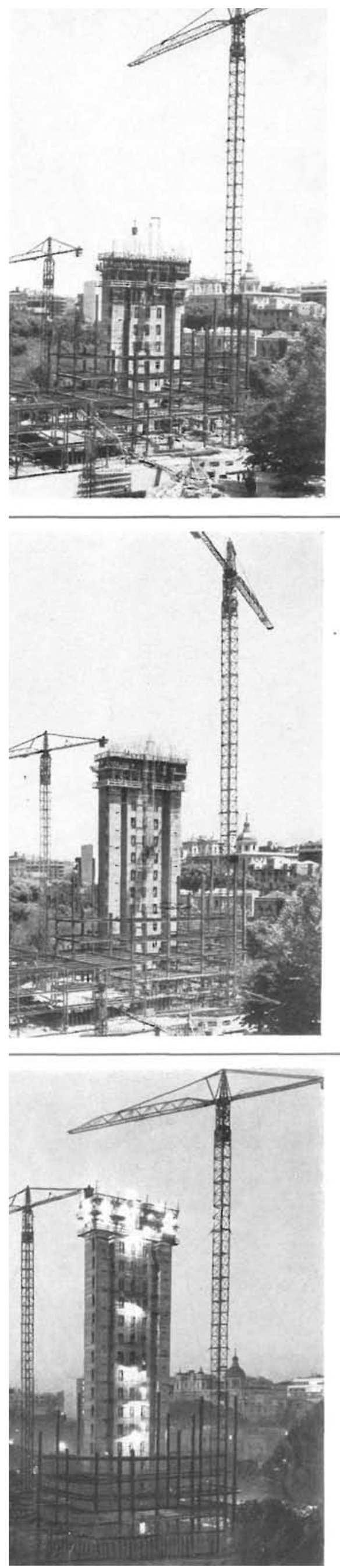
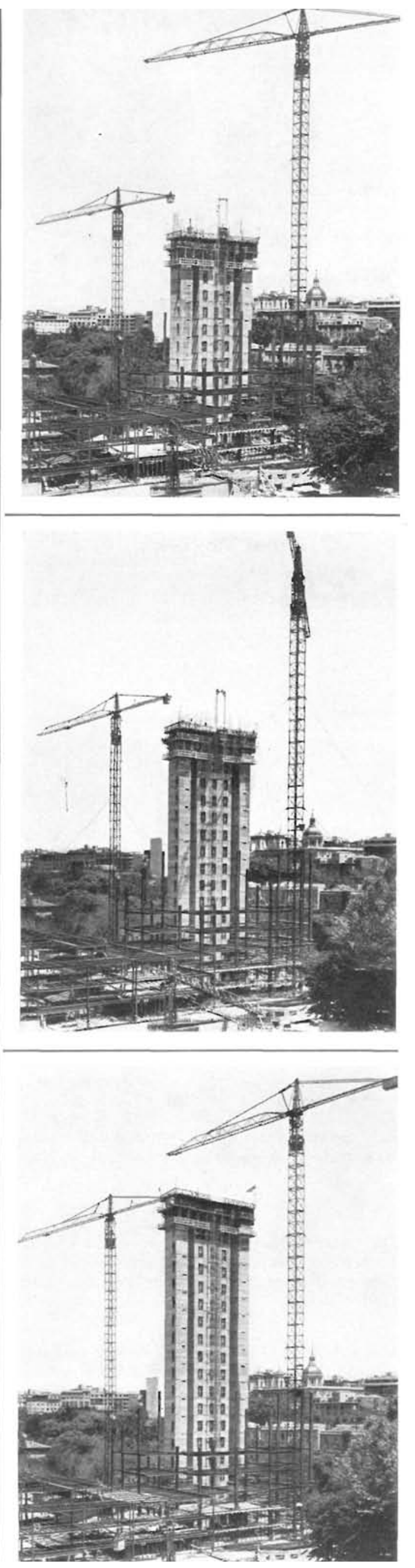

Fases de construcción del núcleo central.

para contrarrestar dicha torsión, se aumentó la capa de compresión de los forjados cerámicos de las distintas plantas a $4 \mathrm{~cm}$, poniendo, además, un mallazo continuo para rigidizarlos.

Este núcleo central, de hormigón, en el que están alojados los ascensores y conductos de aire acondicionado, se ejecutó con encofrado deslizante; el central, desde la cota 0,00 a la 63,00 , y los laterales, por ser su parte inferior de palastro, como antes hemos indicado, desde la 15,00 hasta la 63,00. Se inició el deslizamiento hormigonando exclusivamente el núcleo central hasta la cota 15,00 , en que se realizó el ensamble con los dos núcleos laterales para continuar hormigonando los tres núcleos, siendo la primera vez que se ha utilizado en España este procedimiento para un edificio.

Todos los huecos para puertas y conductos de aire acondicionado, cajetines de ascensores, etc., fueron realizados simultáneamente, dejando los marcos adecuados.

Asimismo, se fueron colocando las placas necesarias para el posterior soldado de la estructura metálica del edificio.

Los núcleos laterales se arriostraron al central mediante estructuras metálicas en forma de cruces de San Andrés.

Se ha empleado el sistema SIEMCRETE, licencia de la casa alemana SIEMENS BAUUNION GMBH, representado en España por TECNES (Técnicas Especiales, S. A.).

\begin{tabular}{l|l}
$20-V I-68$ & $23-V I-68$ \\
\hline $24-V I-68$ & $25-V I-68$ \\
\hline $27-V I-68$ & $28-V I-68$
\end{tabular}


Sus principales características son:

a) gatos oleohidráulicos de $7 \mathrm{Mp}$ de potencia;

b) cabezas de agarre por anillo de acero extraduro;

c) tubos de trepada con carga de rotura de $12 \mathrm{Mp}$;

d) bomba eléctrica con dispositivo de parada automático;

e) tres plataformas de trabajo.

El armado del encofrado deslizante se realizó en una zona adyacente y fue trasladado a los puntos de arranque mediante una grúa-torre. Allí se procedió al montaje final y construcción de las plataformas de trabajo.

Como medios de elevación, se emplearon simultáneamente una grúa-torre P-80 y un ascensor MULTI-LIFT, también de la firma TECNES.

El ascensor se encargó de todo el movimiento de personal y ayudó a la grúa en la elevación de materiales.

La distribución del trabajo se realizó así:

\section{Plataforma superior:}

Distribución del hormigón mediante tolvas, colocación de barras de armadura vertical, y almacenamiento de materiales.

Plataforma intermedia:

Colocación de armadura horizontal, vertido y vibrado del hormigón, colocación de marcos para huecos, y soldadura de placas de anclaje.

\section{Plataforma inferior:}

Montaje de las estructuras metálicas de arriostramiento de los núcleos laterales con el central, fratasado de la superficie, y riego del hormigón.

La rapidez de desplazamiento, la distribución de operarios en las tres plataformas, ausencia de interferencias y el hecho de ser ciclos repetitivos - un ciclo por planta-, permitió obtener un elevado rendimiento de la mano de obra. Para todo ello se preparó un programa de tiempos PERT.

El total de la superficie a deslizar fue de $5.900 \mathrm{~m}^{2}$, y para ello se utilizaron 28 puestos de elevación con dos gatos cada uno, y una separación entre ellos de $2,50 \mathrm{~m}$; el volumen de hormigón por metro de altura fue de $14 \mathrm{~m}^{3}$. Se empezó a deslizar el 14 de junio de 1968, y se terminó el 28 de junio -en total, 14 días-, lo que hace una velocidad media de $4,5 \mathrm{~m}$ por día, siendo la velocidad máxima en un día de 5,05 metros. 


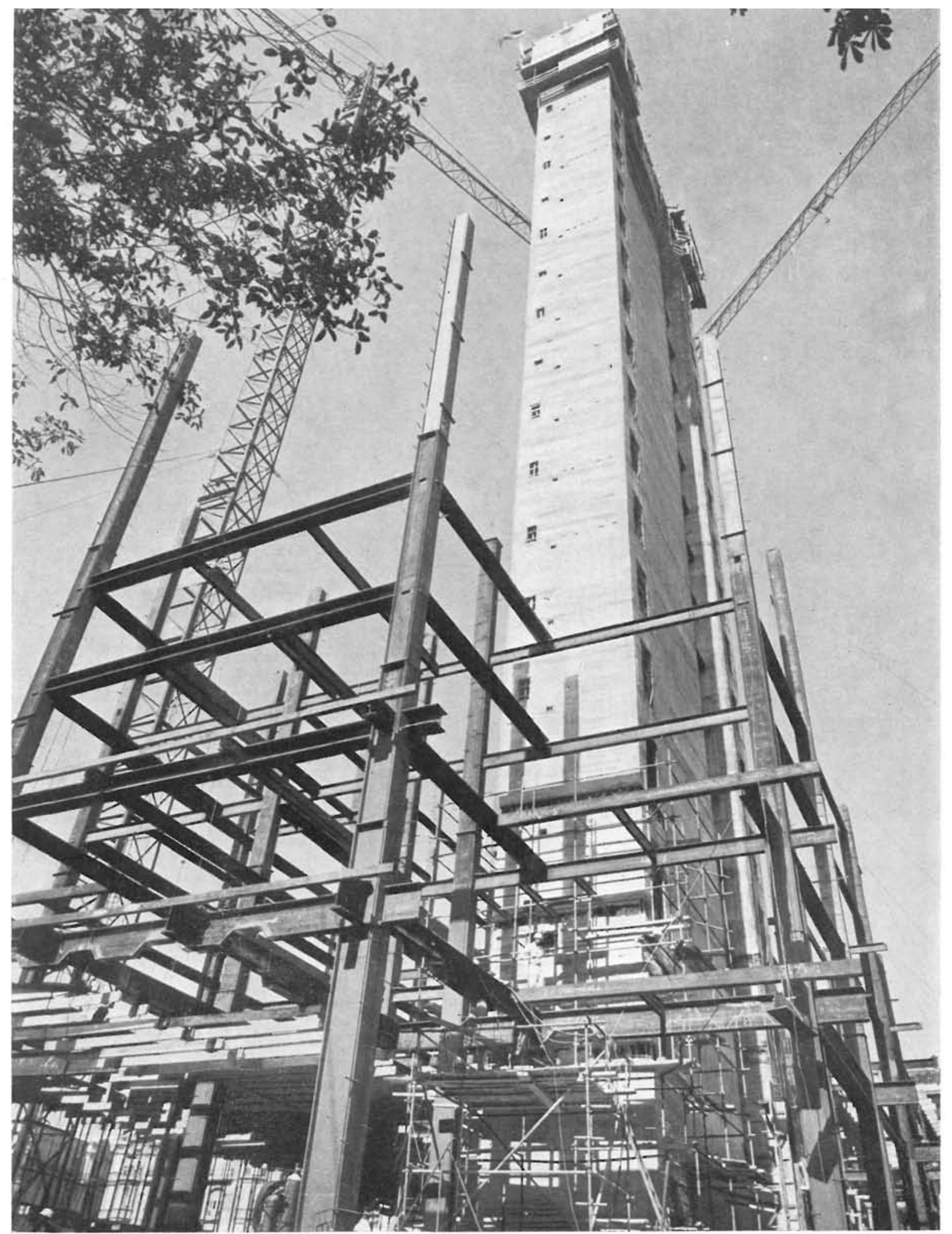

Una vez terminado el deslizamiento, se continuó con la estructura metálica y con los forjados normales, que han sido hormigonados empleando bomba de hormigón de $12 \mathrm{~m}^{3} /$ hora, con hormigón prefabricado, alcanzando la cota máxima de $73 \mathrm{~m}$ (planta 18), que es la altura máxima alcanzada en España con este tipo de máquina.

En la parte posterior de la torre se ha construido un salón de actos capaz para 700 personas, todo él en estructura metálica. 


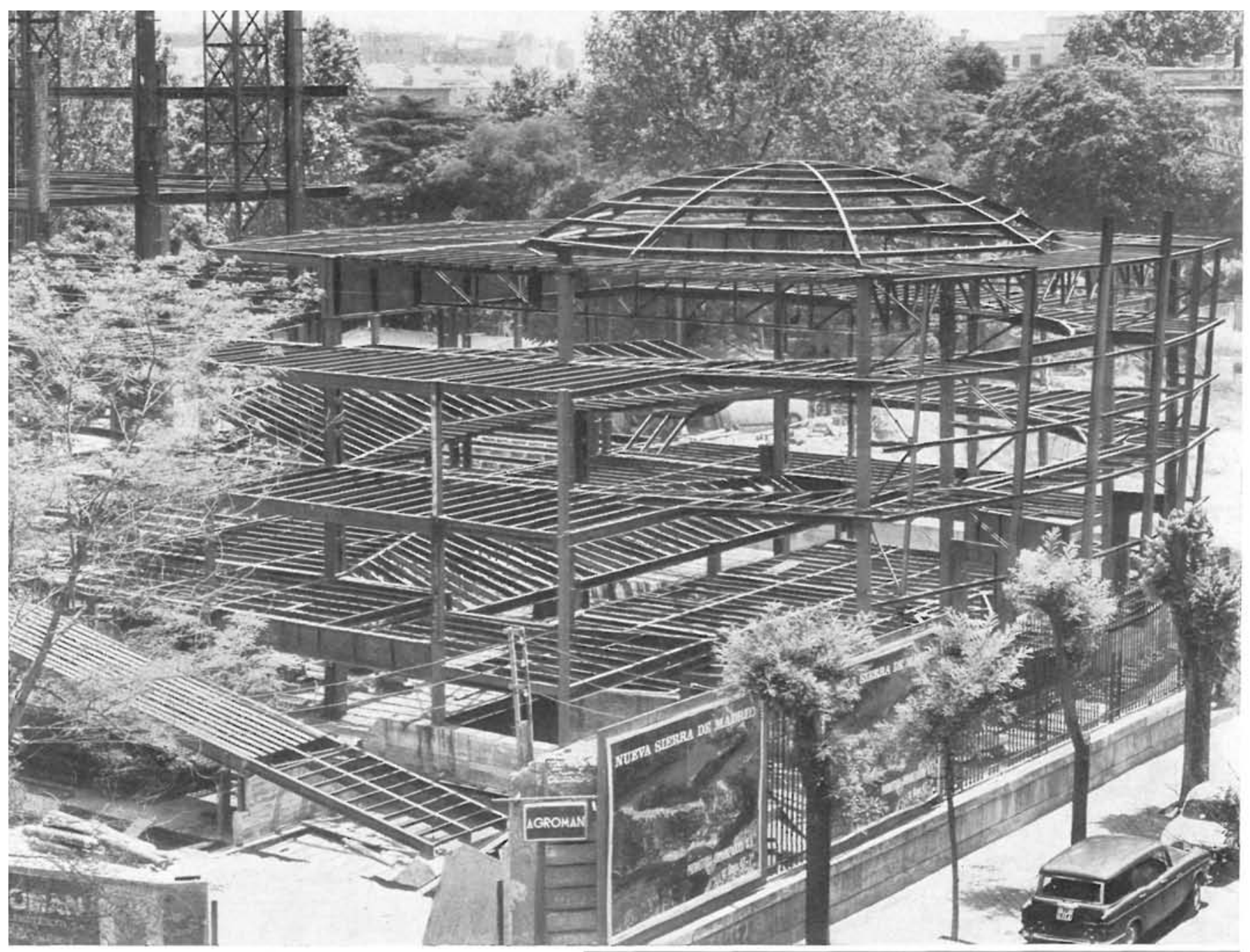

Salón de actos: estructura.

En total se han colocado $2.130 \mathrm{t}$ de hierro en estructura metálica y $12.000 \mathrm{~m}^{3}$ de hormigón.

La obra, en avanzado estado de construcción, tiene terminada la albañilería gruesa, cerrada en fachadas, y está, en etapa de oficios, instalaciones y decoración.

Esta obra ha sido ejecutada por AGROMAN, Empresa Constructora, S. A.

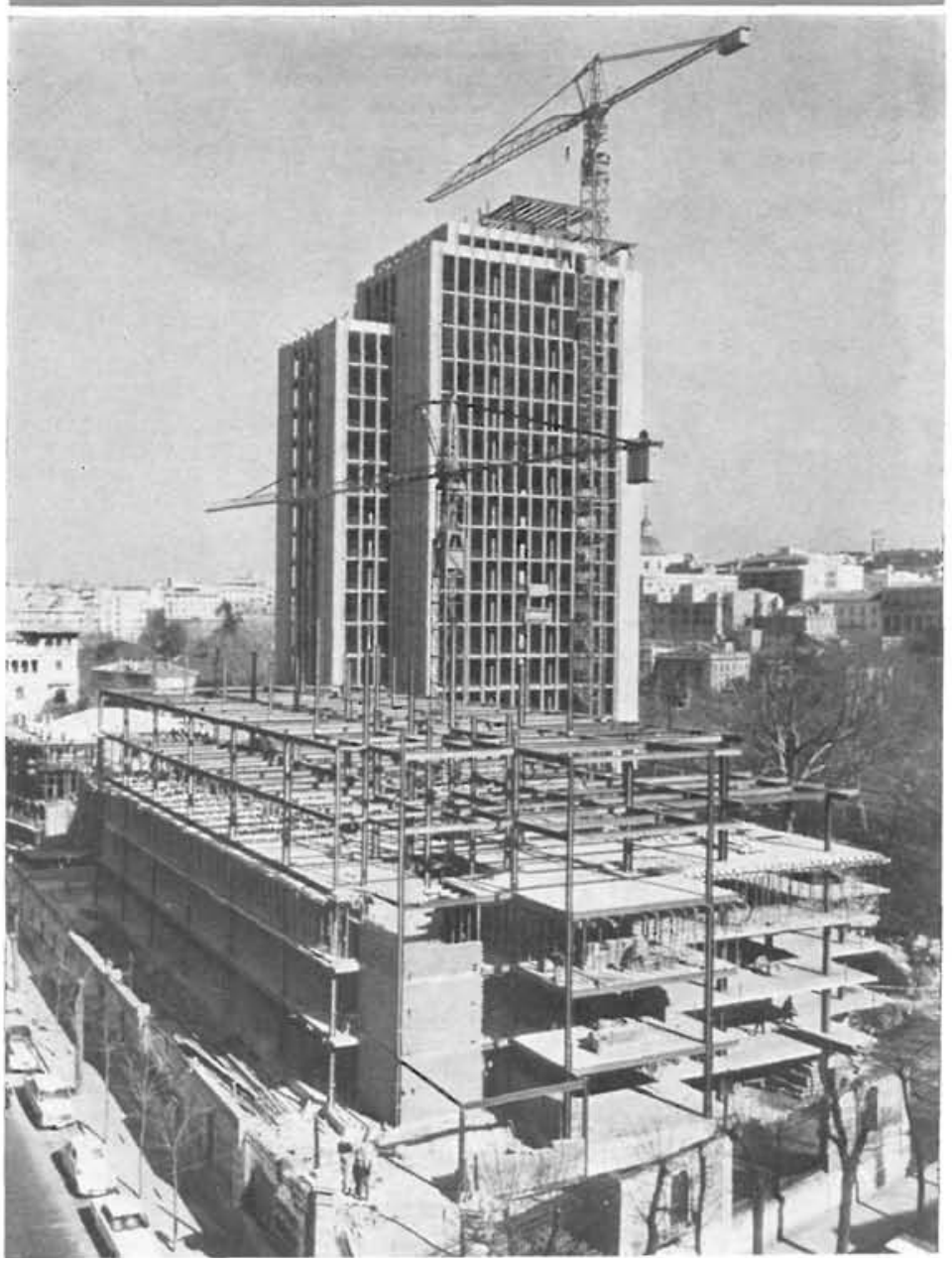




\section{Le comstrmotrion alle l'édiffice pour uLa Unión

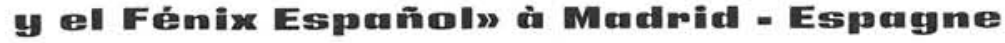

L. Gutiérrez Soto, architecte; J. M.* Sáenz de Benito, V. Urguía, J. Gascón y Marín et J. A. Fernández Durán, ingénieurs

Cet article explique les détails les plus intéressants de la construction de cet édifice, les problèmes posés par les fondations -étant donné les caractéristiques particulières du terrain - et les solutions adoptées. Il décrit également l'exécution spéciale du noyau central des ascenseurs, métallique à sa base et dont le fût a été exécuté à l'aide d'un coffrage glissant.

L'édifice comprend trois niveaux inférieurs et dix-neuf étages.

\section{The Gomstrmuction Process of the utonion y Fenix

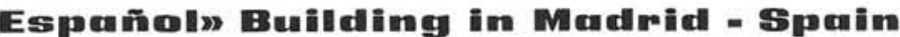

L. Gutiérrez Soto, architect; J. M. Sáenz de Benito, V. Urguía, J. Gascón y Marín and J. A. Fernández Durán, engineers

This article describes the most interesting features in the construction of this tall building. Mention is made of the foundation problems, due to the special nature of the soil, and the solutions adopted. Information is also given of the construction of the central lift shapft, with a steel base, and the rest made with sliding formwork.

The building has three levels below the ground and 19 storeys.

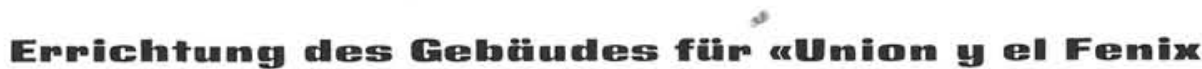

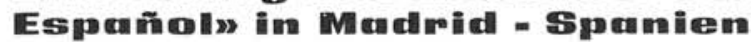

L. Gutiérrez Soto, Architekt; J. M." Sáenz de Benito, V. Urguía, J. Gascón y Marín und J. A. Fernández Durán, Ingenieure

In diesem Artikel werden die interessantesten Details des Baus erklärt, die durch die Baugründung aufgeworfenen Probleme - aufgrund der speziellen Eigenschaften des Geländes- und ihre Lösungen; desgleichen wird auch die besondere Gestaltung des Zentralkerns der Aufzüge beschrieben, dessen unterer Abschnitt aus Metall, der Rest des Schaftes in Kletterschalung ausgeführt ist.

Das Gebäude weist drei Keller- und 19 Nutzgeschosse auf. 